\title{
Enhanced polo-like kinase 1 expression in myelodysplastic syndromes
}

\author{
Kyoung Il Min ${ }^{1}$, Silvia Park ${ }^{1}$, Seung-Hwan Shin ${ }^{2}$, Yong-Rim Kwon ${ }^{3}$, Hye-Joung Kim ${ }^{3}$, Yoo Jin Kim ${ }^{3,4}$ \\ ${ }^{1}$ Division of Hematology, Department of Internal Medicine, Seoul St. Mary's Hematology Hospital, ${ }^{2}$ Department of Hematology, \\ Yeoido St. Mary's Hospital, ${ }^{3}$ Laboratory of Hematological Disease and Immunology, ${ }^{4}$ Leukemia Research Institute, College of \\ Medicine, The Catholic University of Korea, Seoul, Korea
}

p-ISSN 2287-979X / e-ISSN 2288-0011 https://doi.org/10.5045/br.2019.54.2.102 Blood Res 2019;54:102-107.

Received on November 9, 2018 Revised on November 22, 2018 Accepted on November 25, 2018

*This study was supported by Basic Science Research Program through the National Research Foundation of Korea (NRF) funded by the Ministry of Education (2018R1D1A1A02049143).

\section{Correspondence to}

Yoo Jin Kim, M.D., Ph.D.

Division of Hematology, Department of

Internal Medicine, Seoul St. Mary's Hematology Hospital, College of Medicine, The Catholic University of Korea, 222 Banpo-daero, Seocho-gu, Seoul 06591, Korea

E-mail: yoojink@catholic.ac.kr

(C) 2019 Korean Society of Hematology

\section{Background}

Cancer is characterized by uncontrolled cellular proliferation, and Polo-like kinase 1 (PLK1), a key regulator of the cell cycle, is overexpressed in many cancers, including acute leukemia and lymphoma. However, the dynamics of PLK1 transcription in myelodysplastic syndromes (MDS) are unknown. This study aimed to investigate the transcript dynamics of PLK1 and determine its role in the pathophysiology of MDS.

\section{Methods}

PLK1 mRNA obtained from the bone marrow samples of 67 patients with MDS, 16 patients with secondary acute myeloid leukemia (sAML), and 10 healthy controls were analyzed using quantitative real-time PCR and compared according to various clinical parameters.

Results

The median PLK1 expression levels differed slightly, but not significantly, between MDS and sAML patients [661.21 (range, 29.38-8,987.31) vs. 1,462.05 (32.22-5,734.09), respectively], but were significantly higher $(P<0.001)$ than the levels in the healthy controls [19.0 (1.60-49.90)]. Further analyses of $P L K 1$ levels according to the WHO classification of MDS, prognostic risk groups, karyotype risk groups, marrow blast percentage, and depth of cytopenia did not reveal any significant associations. In patients progressing to sAML, PLK1 expression levels differed significantly according to the presence or absence of resistance to hypomethylation treatment $(2,470.58$ vs. $415.98, P=0.03)$.

\section{Conclusion}

PLK1 is upregulated in MDS patients; however, its role in the pathophysiology of MDS is unclear. Gene upregulation in cases with pharmacotherapeutic resistance warrants further investigation.

Key Words Myelodysplastic syndromes, Polo-like kinase 1, Protein-serine-threonine kinases, DNA methylation, Gene expression

\section{INTRODUCTION}

Myelodysplastic syndromes (MDS) are a group of clonal hematological neoplasms characterized by ineffective dysplastic hematopoiesis, peripheral-blood cytopenia, and a high risk of progression to acute myeloid leukemia (AML) [1]. Treatment outcomes differ among MDS patients, with median survival ranging from 6 months to 5 years [2]. For optimal guidance in clinical decisions, various prognostic scoring systems have been developed, such as the
International Prognostic Scoring System (IPSS), Revised International Prognostic Scoring System (IPSS-R), and World Health Organization Classification-Based Prognosis Scoring System (WPSS) [3-5]. According to these scoring systems, extent of cytopenia, percentage of bone marrow blasts, and bone marrow cytogenetics are the 3 primary prognostic factors in MDS. Other prognostic factors, such as marrow fibrosis, flow cytometry parameters, and somatic gene mutations, have been suggested as novel prognostic factors [6].

Genetic mutations are receiving increasing attention as prognostic factors, owing to the recent advent of inexpensive 
genome sequencing platforms [7]. Most of the clinical and pathological features of MDS are believed to result from recurrent somatic genetic lesions [8], and genes such as TP53, RUNX1, ETV6, EZH2, ASXL1, and SRSF2 have been identified as independent risk factors for poor prognosis in MDS [9]. However, mutations have been reported in more than 50 different genes, and none of them could be used as a universal marker for MDS.

Quantitative reverse transcription PCR (qRT-PCR) assays of Wilms tumor gene 1 (WT1) have revealed it as a suitable prognostic marker for MDS [10] because upregulation of WT1 is associated with higher IPSS scores [11]. In addition, WT1 expression in MDS patients one month after hematopoietic stem cell transplantation successfully predicted disease relapse [12]. Collectively, these characteristics indicate that WT1 is a useful marker for minimal residual disease in MDS before and after treatment. Unfortunately, other than $W T 1$, no genetic or molecular markers are currently utilized to indicate disease status either during its natural course or after treatment.

Polo-like kinase 1 (PLK1), a serine/threonine kinase, plays an important role in cell cycle regulation, particularly at the G2/M transition, as well as during mitosis and is encoded by $P L K 1$ [13]. Similar to $W T 1, P L K 1$ is expressed at very low levels in most normal tissues but is overexpressed in solid tumors [14] such as colorectal cancers [15] and non-small cell lung cancer [16]. Furthermore, $P L K 1$ is overexpressed in hematologic malignancies such as AML [17]. Moreover, the $P L K 1$ inhibitors volasertib and rigosertib have been reported as promising chemotherapeutic candidates for myeloid malignancies [18].

However, the role of PLK1 expression in MDS pathophysiology is unknown. Therefore, in this study, we quantified PLK1 mRNA expression levels in bone marrow (BM) samples from patients with MDS and secondary AML evolved from MDS (sAML) and analyzed their expression in association with various clinical parameters of MDS to determine the potential role of PLK1 in MDS.

\section{MATERIALS AND METHODS}

\section{Patients}

Patients who were newly diagnosed with MDS and sAML between March 2009 and March 2012 and who agreed to provide their BM samples for the study were screened, and only those patients whose samples were available for molecular analysis were enrolled. The study was approved by the institutional review board of Seoul St. Mary's Hospital, The Catholic University of Korea, and all patients and healthy controls enrolled in the study provided written informed consent before BM sample collection. Clinical and laboratory data at diagnosis or transformation to sAML and data on MDS treatment were obtained.

\section{Definitions}

MDS was diagnosed according to the 2016 WHO classi- fication [19]. IPSS-R scores were calculated at MDS diagnosis [4]. The extent of cytopenia, including absolute neutrophil count (ANC), hemoglobin levels, and platelet count, and cytogenetic risk groups were classified according to IPSS-R. Treatment responses after hypomethylating therapy (HMT) were assessed according to International Working Group 2006 criteria [20].

\section{Assessment of PLK1 transcript levels}

Total RNA was isolated from $5 \times 10^{6}$ mononuclear cells by using TRIzol Reagent (ThermoFisher Scientific, Waltham, MA, USA). cDNA was synthesized from $1 \mu \mathrm{g}$ of total RNA in a $20 \mu \mathrm{L}$ reaction mixture by using the Reverse Transcriptase kit (ThermoFisher Scientific) according to the manufacturer's instructions. Absolute quantification of the PLK1 transcripts was performed by RT-PCR using TaqMan probes (ThermoFisher Scientific). qRT-PCR analysis was performed in a LightCycler 480 Instrument II (Roche, Basel Schweiz, Switzerland). 18S ribosomal RNA (18S rRNA) transcripts were analyzed as a housekeeping gene. Plasmid standards of known copy number were used for normalization and quantification in all experiments.

\section{Statistical analysis}

The Statistical Package for the Social Sciences (SPSS, version 24.0, Inc., Chicago, IL, USA) was used for all statistical analyses. Continuous variables are presented as the median (range). PLK1 expression levels were compared among the

Table 1. Baseline characteristics of patients with myelodysplastic syndrome (MDS) or secondary acute myeloid leukemia (sAML).

\begin{tabular}{lcc}
\multicolumn{1}{c}{ Characteristics } & MDS & sAML \\
\hline Total N of patients, $N$ & 67 & 16 \\
Age, median (range), yr & $54(16-77)$ & $58.5(23-74)$ \\
Male, N (\%) & $45(67 \%)$ & $7(44 \%)$ \\
Hematologic parameters & & \\
$\quad$ ANC $\left(\times 10^{6} / \mathrm{L}\right)$, & $1,190(0-5,045)$ & $625(41-22,570)$ \\
$\quad$ median (range) & & \\
Hemoglobin (g/dL), & $8.9(5.1-15.9)$ & $9.0(5.4-9.6)$ \\
$\quad$ median (range) & & \\
Platelets $\left(\times 10^{9} / \mathrm{L}\right)$, & $73(5-999)$ & $34(9-159)$ \\
$\quad$ median (range) & & \\
BM blasts $(\%)$ & $3(0-17)$ & $32(20-95)$ \\
WHO classification, N (\%) & & \\
MDS-U & $5(7.5)$ & - \\
SLD & $5(7.5)$ & - \\
MLD & $31(46.3)$ & - \\
EB-1 & $13(19.4)$ & - \\
EB-2 & $13(19.4)$ & - \\
HMT failure, N (\%) & & $6(37.5)$ \\
No & - & $10(62.5)$ \\
Yes & - &
\end{tabular}

Abbreviations: ANC, absolute neutrophil count; $\mathrm{BM}$, bone marrow; $\mathrm{EB}$, excess blasts; $\mathrm{HMT}$, hypomethylating treatment; MDS, myelodysplastic syndrome; MDS-U, MDS unclassifiable; MLD, multilineage dysplasia; SAML, secondary acute myeloid leukemia; SLD, single lineage dysplasia. 
control, MDS, sAML, and MDS subgroups using the Mann-Whitney test for two group comparisons and the Kruskal-Wallis test for multiple group comparisons. A $P$-value of less than 0.05 was used to define statistical significance.

\section{RESULTS}

\section{Baseline characteristics}

Sixty-seven patients with MDS were enrolled as well as 16 sAML patients. In 6 of the sAML patients, sAML evolved from MDS without any treatment, and in 10 of the patients, sAML evolved from MDS after HMT failure. Ten healthy controls were also included.

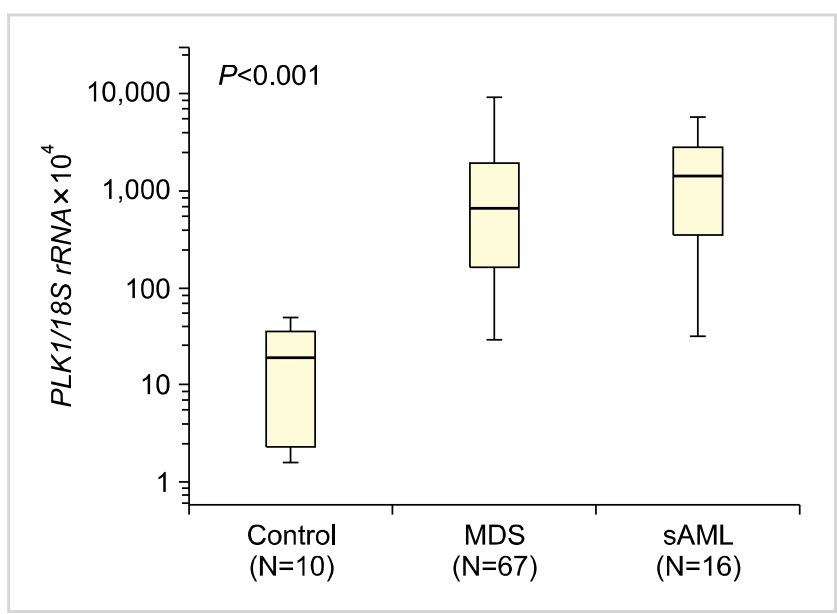

Fig. 1. PLK1 expression levels in healthy controls and patients with MDS and SAML.

Abbreviations: $18 \mathrm{~S}$ rRNA, $18 \mathrm{~S}$ ribosomal RNA; MDS, myelodysplastic syndromes; PLK1, polo-like kinase 1 gene; sAML, secondary acute myeloid leukemia.
Table 1 summarizes the baseline characteristics of the MDS ( $\mathrm{N}=67)$ and sAML $(\mathrm{N}=16)$ patients. The WHO subtypes of MDS were MDS unclassifiable (MDS-U, N=5), MDS with single lineage dysplasia (MDS-SLD, $\mathrm{N}=5$ ), MDS with multilineage dysplasia (MDS-MLD, N=31), MDS with excess blast 1 (MDS-EB-1, N=13), and MDS with excess blast 2 (MDS-EB-2, $\mathrm{N}=13$ ). The most common subtype of MDS was MDS-MLD, which was observed in $46.3 \%$ of the patients, followed by MDS-EB-1 and MDS-EB-2 at 19.4\% each. sAML patients were classified into the natural course group $(\mathrm{N}=6)$ and the HMT failure group $(\mathrm{N}=10)$. The median age was 54 (16-77) years for the MDS patients and 58.5 (23-74) years for the sAML patients. The median percentage of BM blasts was $3 \%$ for MDS patients and 34\% for sAML patients.

\section{PLK1 is overexpressed in MDS and sAML}

We compared the baseline PLK1 expression levels among the healthy controls and MDS and sAML patients. Median PLK1 expression levels differed slightly but not significantly between MDS and sAML patients [661.21 (range, 29.38$8,987.31$ ) vs. $1,462.05$ (32.22-5,734.09), respectively], but were significantly higher than that of the healthy controls [19.0 (1.60-49.90), $P<0.001$; Fig. 1].

\section{PLK1 expression levels in MDS subtypes}

$P L K 1$ expression levels in the MDS patients were evaluated according to WHO subtype. PLK1 expression levels were slightly but not significantly elevated in the MDS-U, MDS-SLD, MDS-MLD, MDS-EB-1, and MDS-EB-2 groups [176.11 (29.38-3,305.33), 708.77 (642.74-3,672.0), 800.37 (96.05-8,541.79), 217.46 (42.51-3,647.54), and 353.15 (75.648,987.31), respectively Fig. 2A].

PLK1 expression was also analyzed according to IPSS-R risk group. Owing to the small number of cases, the very-low-risk and low-risk groups were combined for the
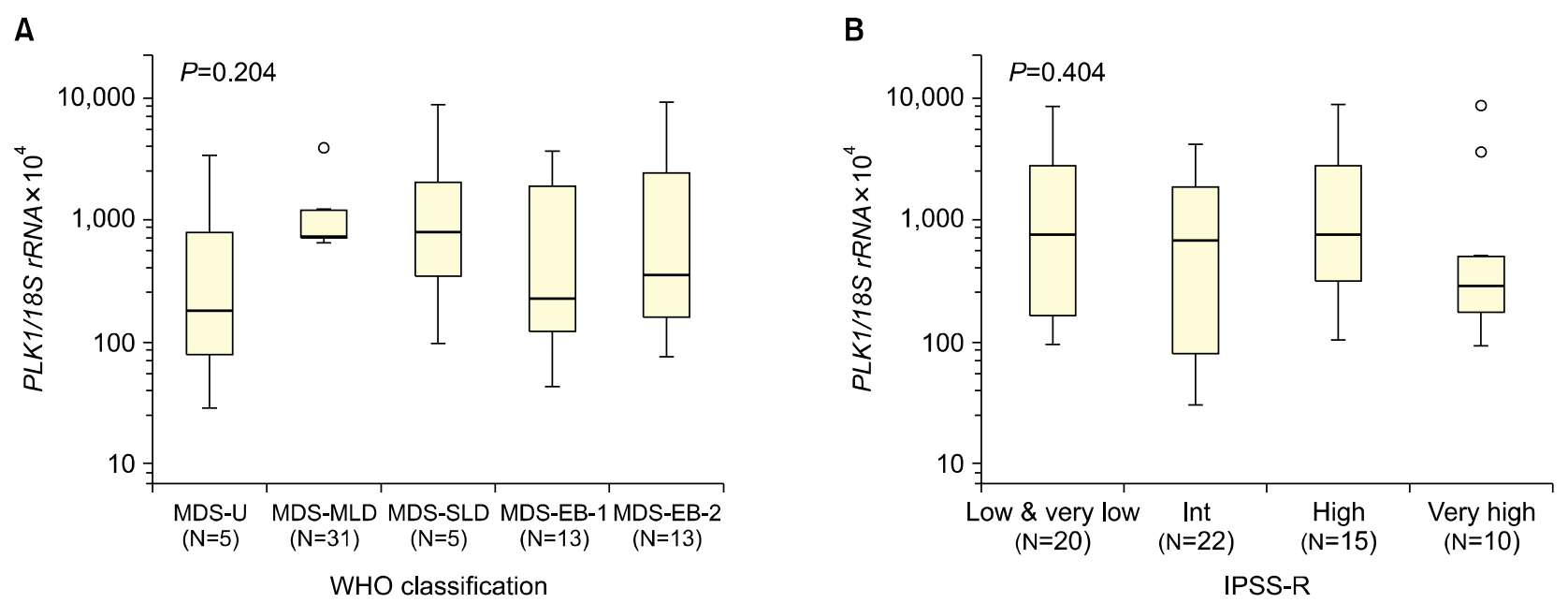

Fig. 2. PLK1 expression levels in MDS patients according to WHO classification (A) and IPSS-R (B).

Abbreviations: 18S rRNA, 18S ribosomal RNA; Int, intermediate; IPSS-R, Revised International Prognostic Scoring System; PLK1, polo-like kinase 1 gene; MDS, myelodysplastic syndromes; MDS-EB-1, MDS with excess blast 1; MDS-EB-2, MDS with excess blast 2; MDS-SLD, MDS with single lineage dysplasia; MDS-MLD, MDS with multilineage dysplasia; MDS-U, MDS unclassifiable; WHO, World Health Organization. 
statistical analysis. The very-low-risk and low-risk, intermediate-risk, high-risk, and very-high-risk groups had PLK1 expression levels of 749.27 (96.05-8,541.79), 684.80 (29.34,179.19), 736.43 (101.1-8,987.31), and 285.31 (93.95$8,715.42)$, respectively, and there were no significant differences among the groups (Fig. 2B). Overall, PLK1 expression levels were not significantly associated with either WHO subtypes or the IPSS-R risk groups.

\section{Association between PLK1 expression levels and the prognostic factors of MDS}

Since $P L K 1$ expression did not differ significantly among WHO subtypes and IPSS-R risk groups, we next investigated the association between $P L K 1$ expression and individual prognostic factors of MDS. For BM blasts, the PLK1 expression levels among patients with blast percentages of $\leq 2$, 2.1-4.9, 5-10, and >10 were 791.21 (78.82-8,541.79), 536.97 (29.38-2,087.15), 189.44 (42.51-3,647.54), and 440.83 (93.95$8,987.31)$, respectively, and they did not differ significantly (Fig. 3A).

Patients were divided into 5 groups according to IPSS-R cytogenetic categories: very good, good, intermediate, poor, and very poor, which showed PLK1 expression levels of 393.68 (78.60-708.77), 736.43 (29.38-8,987.31), 693.85 (75.648,541.79), $334.06(141.12-5,425.64)$, and 520.45 (320.37-
8,715.42), respectively, and there were no significant differences among these groups (Fig. 3B).

Hematologic parameters, such as ANC, hemoglobin, and platelet count, were evaluated separately. PLK1 expression in patients with an ANC $>800 \times 10^{6} / \mathrm{L}$ was $694.04(32.22$ $7,326.63)$, and that in patients with an ANC $<800 \times 10^{6} / \mathrm{L}$ was 653.97 (29.38-8,987.31), and no significant difference was observed (Fig. 3C). PLK1 expression in patients with hemoglobin levels $\geq 10 \mathrm{~g} / \mathrm{dL}, 8-9.9 \mathrm{~g} / \mathrm{dL}$, and $<8 \mathrm{~g} / \mathrm{dL}$ were 296.66 (42.51-7,326.63), 795.07 (29.38-8,987.31), and 749.80 (78.60-5,425.64), respectively. Although PLK1 expression levels tended to increase with a reduction in hemoglobin levels, no significant differences were observed (Fig. 3D). PLK1 expression in patients with platelet counts of $>$ $100 \times 10^{6} / \mathrm{L}, \quad 50-99 \times 10^{6} / \mathrm{L}$, and $<50 \times 10^{6} / \mathrm{L}$ were $1,112.45$ (112.50-8,541.749), 176.11 (29.38-8,987.31), and 560.07 (101.10-8,715.42), respectively. Although the expression levels differed significantly among these 3 groups, they showed neither an increasing nor a decreasing trend in PLK1 expression (Fig. 3E).

\section{PLK1 expression levels in SAML}

$P L K 1$ expression was not associated with an increased risk of MDS, and $P L K 1$ expression in sAML did not differ from that in MDS (Fig. 1). PLK1 expression in sAML patients
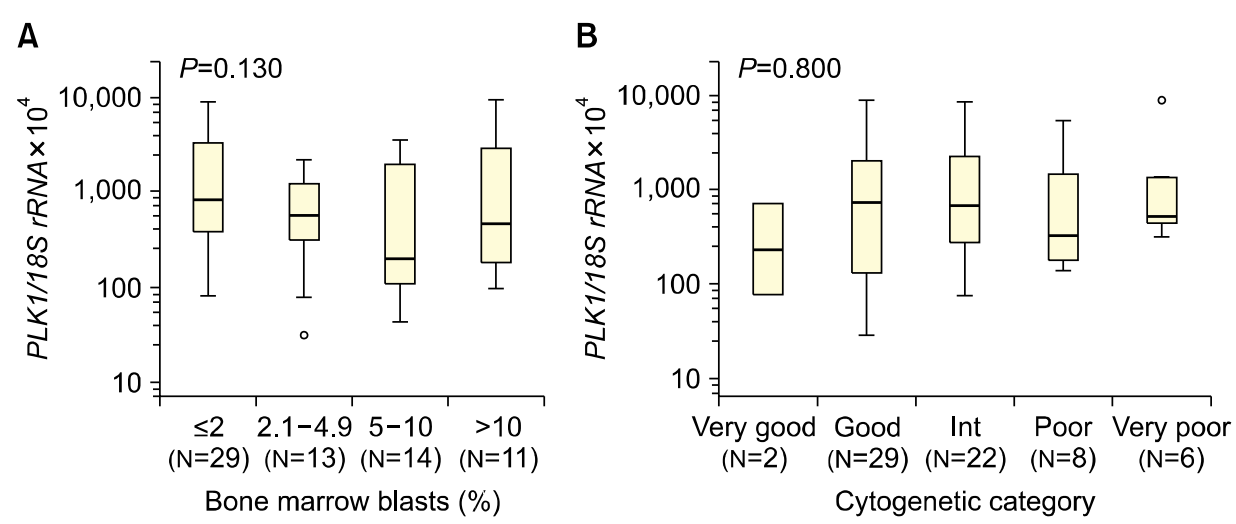

C

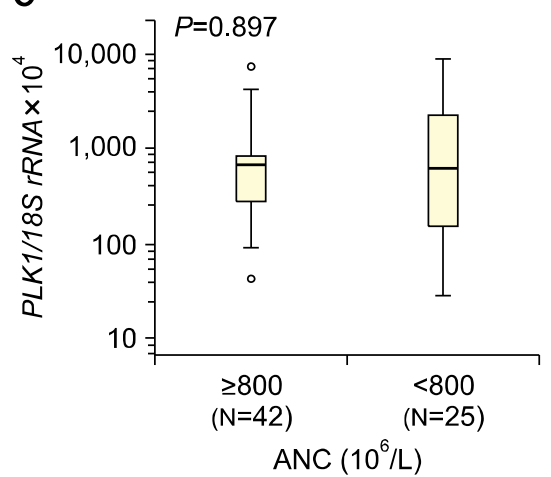

D

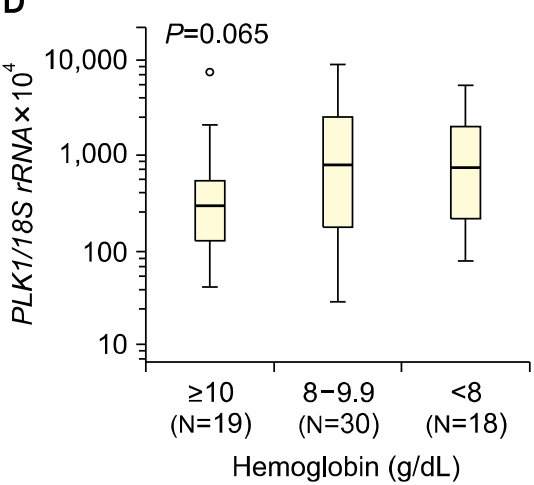

E

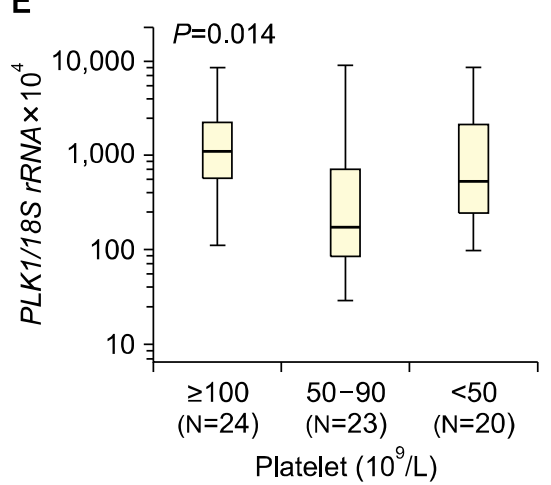

Fig. 3. PLK1 expression levels in MDS patients according to prognostic factors: Bone marrow blast percentage (A), IPSS-R cytogenetic categories (B), absolute neutrophil count (C), hemoglobin level (D), and platelet count (E).

Abbreviations: 18S rRNA, 18 S ribosomal RNA; ANC, absolute neutrophil count; Int, intermediate; IPSS-R, Revised International Prognostic Scoring System; MDS, myelodysplastic syndromes; PLK1, polo-like kinase 1 gene; sAML, secondary acute myeloid leukemia. 


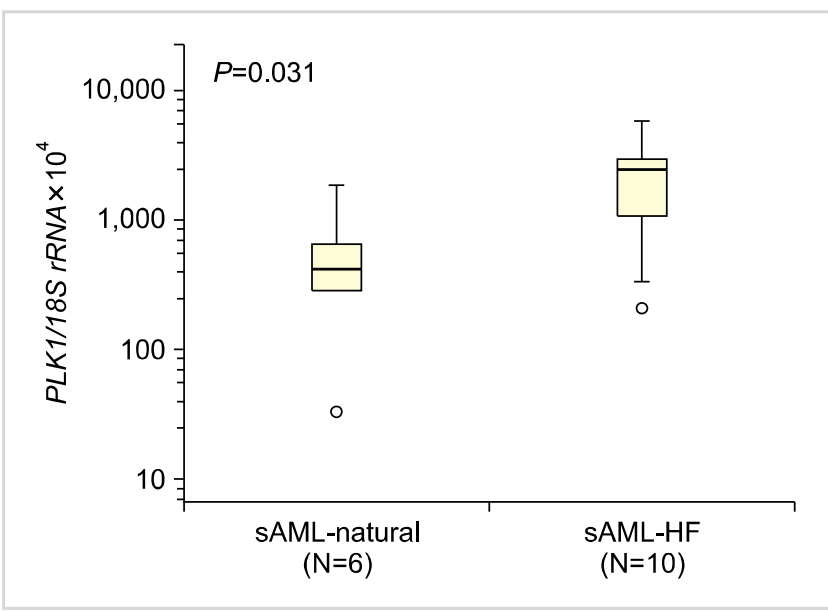

Fig. 4. PLK1 expression levels in patients with SAML according to whether the disease developed "naturally" or after hypomethylating treatment failure.

Abbreviations: $18 \mathrm{~S}$ rRNA, $18 \mathrm{~S}$ ribosomal RNA; PLK1, polo-like kinase 1 gene; sAML, secondary acute myeloid leukemia; sAML-HF, patients who developed secondary acute myeloid leukemia after hypomethylating treatment failure; sAML-natural, patients who developed secondary acute myeloid leukemia with no prior disease-modifying treatment.

was analyzed based on prior HMT. The median PLK1 expression level in sAML patients with disease progression due to HMT failure (sAML-HF) was 2,470.58 (214.01$5,734.09$ ), which was significantly higher than that in the sAML group without prior disease-modifying treatment [sAML-natural; 415.98 (32.22-1,850.46); $P=0.03$, Fig. 4], suggesting that $P L K 1$ expression levels are associated with HMT resistance.

\section{DISCUSSION}

PLK1 encodes polo-like kinase 1, a serine/threonine protein kinase that plays a key regulatory role in mitosis and cytokinesis [13]. The gene is located on chromosome 16p12, and it was originally identified as polo, the founding member of the PLK family, a protein that plays an essential role in delicate mitotic events in Drosophila melanogaster [21]. Five mammalian PLKs have been identified, and PLK1 is the most extensively characterized [18]. Generally, PLK1 is expressed at low levels during G0/G1 phase, gradually increases during $\mathrm{S}$ phase, and then peaks during $\mathrm{M}$ phase [22].

Loss of control over cellular proliferation is a hallmark of cancer [23]. Owing to its essential roles in multiple steps of mitosis and the cellular responses to DNA damage and replication stress [24], PLK1 has been investigated in many different cancers. PLK1 is overexpressed in solid tumors such as lung cancers and colorectal cancers, and its expression is increased at higher stages of cancer $[15,16]$. In some tumor types, $P L K 1$ overexpression correlates with a worse prognosis. $P L K 1$ upregulation has also been observed in hematologic malignancies such as AML, acute lymphoblastic leukemia, and diffuse large B-cell lymphoma [17, 25, 26]. Despite these observations, no study has elucidated the role of $P L K 1$ in the pathogenesis or progression of MDS.

In the present study, PLK1 mRNA was overexpressed in the BM mononuclear cells of patients with MDS compared to the expression levels in healthy controls, suggesting that $P L K 1$ is associated with MDS pathophysiology. However, $P L K 1$ expression levels did not differ significantly between MDS and sAML patients, contrary to our speculation that PLK1 upregulation and the resultant higher mitotic activity should be observed when the disease transforms to AML. Moreover, the percentage of BM blasts was not associated with $P L K 1$ expression levels, as the percentages were similar among the groups of MDS patients classified based on WHO subtypes, suggesting that $P L K 1$ overexpression does not simply reflect fast proliferating cancer cells and is not directly responsible for the progression of MDS. Similar findings were also observed when we compared PLK1 expression levels according to risk groups via the IPSS-R and to the risk for each parameter in the IPSS-R system, such as the depth of cytopenia and karyotype. These data suggest that PLK1 is probably associated with MDS pathogenesis; however, its role in the pathophysiology or progression of MDS could be quite different from that of WT1 in MDS [11].

Interestingly, patients who progressed from MDS to SAML after HMT failure showed remarkably higher PLK1 expression levels than those who progressed to SAML without prior HMT. However, it is unknown whether upregulation of $P L K 1$ is a cause or result of HMT resistance. Furthermore, the number of sAML cases in this study was too small to draw any conclusive association between PLK1 expression levels and HMT resistance. However, the role of PLK1 in HMT resistance warrants further investigation. Analysis of PLK1 transcript levels both pre- and post-HMT could reveal its feasibility as a biomarker for HMT response or resistance [27]. Mechanistic information regarding PLK1 and HMT resistance could explain why rigosertib, a PLK1 inhibitor, did not significantly improve overall survival in high-risk MDS patients with HMT failure when compared with the best supportive care [28]. In addition, an understanding of the underlying mechanism would facilitate the introduction of various PLK1 inhibitors [29].

To the best of our knowledge, this is the first study to investigate the role of PLK1 in MDS, even though it has several limitations, including the relatively small number of patients enrolled and the fact that PLK1 expression levels were not compared between HMT-resistant and -responsive patients after HMT. Furthermore, PLK1 dynamics were not investigated during or after HMT in association with primary or secondary resistance. Based on the present results, we intend to investigate the molecular association between HMT and $P L K 1$ expression.

In conclusion, $P L K 1$ was upregulated in MDS and SAML; however, the association between PLK1 expression levels and MDS risk factors is not yet clear. The difference in PLK1 expression levels in sAML patients according to the presence or absence of HMT resistance suggest the need for future studies on the role of PLK1 in HMT resistance. 


\section{Authors' Disclosures of Potential Conflicts of Interest}

No potential conflicts of interest relevant to this article were reported.

\section{REFERENCES}

1. Adès L, Itzykson R, Fenaux P. Myelodysplastic syndromes. Lancet 2014;383:2239-52.

2. Gangat N, Patnaik MM, Tefferi A. Myelodysplastic syndromes: contemporary review and how we treat. Am J Hematol 2016; 91:76-89.

3. Greenberg P, Cox C, LeBeau MM, et al. International scoring system for evaluating prognosis in myelodysplastic syndromes. Blood 1997;89:2079-88.

4. Greenberg PL, Tuechler H, Schanz J, et al. Revised international prognostic scoring system for myelodysplastic syndromes. Blood 2012;120:2454-65.

5. Malcovati L, Germing U, Kuendgen A, et al. Time-dependent prognostic scoring system for predicting survival and leukemic evolution in myelodysplastic syndromes. J Clin Oncol 2007;25: 3503-10.

6. Fenaux $P$, Adès L. How we treat lower-risk myelodysplastic syndromes. Blood 2013;121:4280-6.

7. Steensma DP, Bejar R, Jaiswal S, et al. Clonal hematopoiesis of indeterminate potential and its distinction from myelodysplastic syndromes. Blood 2015;126:9-16.

8. Sperling AS, Gibson CJ, Ebert BL. The genetics of myelodysplastic syndrome: from clonal haematopoiesis to secondary leukaemia. Nat Rev Cancer 2017;17:5-19.

9. Bejar R, Stevenson K, Abdel-Wahab O, et al. Clinical effect of point mutations in myelodysplastic syndromes. N Engl J Med 2011;364 2496-506.

10. Galimberti S, Ghio F, Guerrini F, et al. WT1 expression levels at diagnosis could predict long-term time-to-progression in adult patients affected by acute myeloid leukaemia and myelodysplastic syndromes. Br J Haematol 2010;149:451-4.

11. Cilloni D, Gottardi E, Messa F, et al. Significant correlation between the degree of WT1 expression and the International Prognostic Scoring System Score in patients with myelodysplastic syndromes. J Clin Oncol 2003;21:1988-95.

12. Yoon JH, Jeon YW, Yahng SA, et al. Wilms tumor gene 1 expression as a predictive marker for relapse and survival after hematopoietic stem cell transplantation for myelodysplastic syndromes. Biol Blood Marrow Transplant 2015;21:460-7.

13. Barr FA, Silljé HH, Nigg EA. Polo-like kinases and the orchestration of cell division. Nat Rev Mol Cell Biol 2004;5:429-40.

14. Eckerdt F, Yuan J, Strebhardt K. Polo-like kinases and oncogenesis. Oncogene 2005;24:267-76.

15. Takahashi T, Sano B, Nagata T, et al. Polo-like kinase 1 (PLK1) is overexpressed in primary colorectal cancers. Cancer Sci 2003; 94:148-52.

16. Wolf G, Elez R, Doermer A, et al. Prognostic significance of polo-like kinase (PLK) expression in non-small cell lung cancer. Oncogene 1997;14:543-9.

17. Renner AG, Dos Santos C, Recher C, et al. Polo-like kinase 1 is overexpressed in acute myeloid leukemia and its inhibition preferentially targets the proliferation of leukemic cells. Blood 2009;114:659-62.

18. Talati C, Griffiths EA, Wetzler M, Wang ES. Polo-like kinase inhibitors in hematologic malignancies. Crit Rev Oncol Hematol 2016;98:200-10.

19. Arber DA, Orazi A, Hasserjian R, et al. The 2016 revision to the World Health Organization classification of myeloid neoplasms and acute leukemia. Blood 2016;127:2391-405.

20. Cheson BD, Greenberg PL, Bennett JM, et al. Clinical application and proposal for modification of the International Working Group (IWG) response criteria in myelodysplasia. Blood 2006; 108:419-25

21. Strebhardt K. Multifaceted polo-like kinases: drug targets and antitargets for cancer therapy. Nat Rev Drug Discov 2010;9: 643-60.

22. Ikezoe T, Yang J, Nishioka C, et al. A novel treatment strategy targeting polo-like kinase 1 in hematological malignancies. Leukemia 2009;23:1564-76.

23. Hanahan D, Weinberg RA. Hallmarks of cancer: the next generation. Cell 2011;144:646-74.

24. Degenhardt Y, Lampkin T. Targeting Polo-like kinase in cancer therapy. Clin Cancer Res 2010;16:384-9.

25. Liu L, Zhang M, Zou P. Expression of PLK1 and survivin in diffuse large B-cell lymphoma. Leuk Lymphoma 2007;48:2179-83.

26. Hartsink-Segers SA, Exalto C, Allen M, et al. Inhibiting Polo-like kinase 1 causes growth reduction and apoptosis in pediatric acute lymphoblastic leukemia cells. Haematologica 2013;98:1539-46.

27. Jung SH, Kim YJ, Yim SH, et al. Somatic mutations predict outcomes of hypomethylating therapy in patients with myelodysplastic syndrome. Oncotarget 2016;7:55264-75.

28. Garcia-Manero G, Fenaux P, Al-Kali A, et al. Rigosertib versus best supportive care for patients with high-risk myelodysplastic syndromes after failure of hypomethylating drugs (ONTIME): a randomised, controlled, phase 3 trial. Lancet Oncol 2016;17: 496-508.

29. Palmisiano ND, Kasner MT. Polo-like kinase and its inhibitors: Ready for the match to start? Am J Hematol 2015;90:1071-6. 